\title{
An Extremal Problem \\ Related to the Maximum Modulus Theorem for Stokes Functions
}

\author{
W. Kratz
}

Abstract. There are considered classical solutions $v$ of the Stokes system in the ball $B=$ $\left\{x \in \mathbb{R}^{n}:|x|<1\right\}$, which are continuous up to the boundary. We derive the optimal constant $c=c_{n}$ such that, for all $x \in B$,

$$
|v(x)| \leq c \max _{\xi \in \theta B}|v(\xi)|
$$

holds for all such functions. We show that $c_{n}=\max _{x \in B} c_{n}(x)$ exists, where $c_{n}(x)$ is the minimal constant in $(*)$ for any fixed $x \in B$. The constants $c_{n}(x)$ are determined explicitly via the Stokes-Poisson integral formula and via a general theorem on the norm of certain linear mappings given by some matrix kernel. Moreover, the asymptotic behaviour of the $c_{n}(x)$ as $x \rightarrow \partial B$ and as $n \rightarrow \infty$ is derived.

In the concluding section the general result on the norm of linear mappings is used to prove two inequalities: one for linear combinations of Fourier coefficients and the other from matrix analysis.

Keywords: Stokes system, maximum modulus theorem, Stokes-Poisson integral formula, norm of linear mappings

AMS subject classiflcation: 35 Q 30, 76 D 07, 47 A 30, 15 A 45

\section{Introduction}

In this paper we consider classical solutions of the Stokes system, so-called Stokes functions, i.e., functions $v: \Omega \rightarrow \mathbb{R}^{n}$ for some domain $\Omega \subset \mathbb{R}^{n}(n \in \mathbb{N})$ such that

$$
v \in C^{2}(\Omega) \text {, and there exists a "pressure function" } p \in C^{1}(\Omega),
$$

such that $(v, p)$ solves the Stokes system in $\Omega$, i.e.,

$$
\Delta v=\operatorname{grad} p, \operatorname{div} v \text { is constant in } \Omega \text {. }
$$

Observe that we require only that $\operatorname{div} v$ is constant rather than zero as usual.

Stokes functions do not satisfy a maximum principle like harmonic functions. But there exist so-called maximum modulus theorems, which state that the modulus of

W. Kratz: Universität Ulm, Abteilung Mathematik V, D - 89069 Ulm 
Stokes functions in a bounded domain is majorized by the maximum of their modulus on the boundary times a certain constant larger than one depending on the domain (see [6], [7: Theorems 2.1 and 2.2] and [8]). In a recent paper [3] there is derived a maximum modulus theorem for Stokes functions in a ball with an explicit constant (in contrast to the cited papers), more precisely:

If $v$ is a Stokes function in a ball $\Omega=B_{r}\left(x_{0}\right)=\left\{x \in \mathbb{R}^{n}:\left|x-x_{0}\right|<r\right\}$, which is continuous up to the boundary, then for all $x \in \Omega$,

$$
|v(x)| \leq c \max _{\xi \in \partial \Omega}|v(\xi)|
$$

for $c=\frac{n(n+1)}{2}(\partial \Omega$ denotes the boundary of $\Omega)$. Note that the pressure $p$ does not occur in the inequality like in other estimates (see, e.g., [2] or [10]).

The constant $c=\frac{n(n+1)}{2}$ is not optimal for $n \geq 2$, but it must be greater or equal to $n$ (see [3: Remark 3]). In this paper we study the "extremal problem" to determine the -best constant denoted by $c_{n}$. Thus, $n \leq c_{n} \leq \frac{n(n+1)}{2}$, and in the plane we have the improved inequality $2<c_{2}<\sqrt{5}$ (see [4: Formula (13)]). Moreover, for any fixed $x \in \Omega=B_{r}\left(x_{0}\right)$, we will determine the best constant $c_{n}(x)$, for which $(*)$ holds. Hence, $c_{n}=\sup _{x \in \Omega} c_{n}(x)$. Since $v\left(r x+x_{0}\right)$ is a Stokes function in $B=B_{1}(0)$ whenever $v$ solves the Stokes system in $B_{r}\left(x_{0}\right)$, we assume throughout that $\Omega=B$. Therefore we consider the quantities

$$
c_{n}(x):=\sup \left\{|v(x)| \mid \begin{array}{l}
v \cdot \text { is a Stokes function in } B \text { with } \\
v \in C(B \cup \partial B) \text { such that } \max _{\xi \in \partial B}|v(\xi)| \leq 1
\end{array}\right\}
$$

where

$$
B:=\left\{x \in \mathbb{R}^{n}:|x|<1\right\} .
$$

Our derivation of $c_{n}(x)$ is based on the Stokes-Poisson integral formula [3: Theorem 1], which states that a Stokes function $v$ in the unit ball $B$ with continuous boundary. values is given by (for $n \geq 2$ )

$$
v(x)=\int_{\partial B} S(\xi, x) v(\xi) d \sigma(\xi) \quad \text { for } x \in B,
$$

where the Stokes kernel $S(\xi, x)$ is an explicitly given symmetric $(n \times n)$-matrix for $(\xi, x) \in \partial B \times B$ (see formula (6) below). It follows from the general result (Theorem 1 ) of the next section that

$$
c_{n}(x)=\max _{|\delta|=1} \int_{\partial B}|S(\xi, x) \delta| d \sigma(\xi) \quad \text { for } x \in B .
$$

We show that $c_{n}=\max _{x \in B} c_{n}(x)$ exists, and we conjecture that

$$
c_{n}=c_{n}(0)=\frac{n \Gamma(n / 2)}{\sqrt{\pi} \Gamma((n-1) / 2)} \int_{0}^{\pi / 2}(\sin \varphi)^{n-2}\left\{1+n(n+2) \cos ^{2} \varphi\right\}^{1 / 2} d \varphi
$$


for all $n \geq 2$. This conjecture is true for $n=2$, so that

$$
2<c_{2}=\frac{2}{\pi} \int_{0}^{\pi / 2} \sqrt{1+8 \cos ^{2} \varphi} d \varphi=2.127 \ldots<\sqrt{5}
$$

which is an elliptic integral. This result is shown in the paper [5]. Numerical calculations by $A$. Peyerimhoff for dimensions $n=3,4,5,6$ support our conjecture, but so far there is no rigorous proof for any $n \geq 3$.

The paper is organized as follows. In the next Section 2 we derive a general theorem, which leads to formula (**). In Section 3 we discuss the Stokes kernel. In particular we show that it is an invertible matrix for all $x \in B$ and $\xi \in \partial B$. This property is certainly of interest for itself. In Section 4 we show formula $(* *)$, and we derive the asymptotic behaviour of $c_{n}(x)$ as $|x| \rightarrow 1-$ and of $c_{n}(0)$ as $n \rightarrow \infty$. Finally, in the last Section 5 , the general result of Section 2 is used to establish two inequalities.

\section{The general theorem}

In this section we solve the following general extremal problem: determine the norm of a linear mapping given by some matrix kernel.

Theorem 1. Let be given a measure space $(\Omega, \Sigma, \mu)$ and a matrix-valued function $K: \Omega \rightarrow \mathbb{C}^{n \times n}$ with matrix elements

$$
k_{\mu \nu} \in L^{1}(\Omega) \quad \text { for } 1 \leq \mu, \nu \leq n
$$

Then

$$
\|\ell\|:=\sup \left\{|\ell(v)|:\|v\|_{\infty} \leq 1\right\}=\max \{f(\delta):|\delta|=1\}
$$

where the linear mapping $\ell: L^{\infty}(\Omega) \rightarrow \mathbb{C}^{n}$ is given by

$$
\ell(v)=\int_{\Omega} K(\omega) v(\omega) d \mu(\omega)
$$

and where the function $f: \mathbb{C}^{n} \rightarrow \mathbb{R}$ is defined by

$$
f(\delta):=\int_{\Omega}\left|K^{*}(\omega) \delta\right| d \mu(\omega)
$$

Throughout the following notation is used: $|x|$ denotes the Euclidean norm of vectors $x \in \mathbb{C}^{n}$ and $\|A\|=\max \{|A x|:|x| \leq 1\}$ the induced matrix norm (i.e., the spectral norm). $\cdots$ As usual $\|v\|_{\infty}:=$ ess $\sup \{|v(\omega)|: \omega \in \Omega\}$ is the-essential supremum of the Euclidean norm of functions $v: \Omega \rightarrow \mathbb{C}^{n}, v \in L^{\infty}(\Omega)$ if $\|v\|_{\infty}<\infty$, and $A^{*}:=\bar{A}^{T}$ denotes the Hermitian adjoint of matrices (or vectors) $A$. Note that always $\|A\|=\left\|A^{*}\right\|$. 
Proof of Theorem 1. By assumption (3),

$$
c_{1}:=\int_{\Omega}\|K(\omega)\| d \mu(\omega)=\int_{\Omega}\left\|K^{*}(\omega)\right\| d \mu(\omega)<\infty .
$$

Hence, by definition (5), $\left|f\left(\delta_{1}\right)-f\left(\delta_{2}\right)\right| \leq c_{1}\left|\delta_{1}-\delta_{2}\right|$ for $\delta_{1}, \delta_{2} \in \mathbb{C}^{n}$. Therefore $f$ is continuous on $\mathbb{C}^{n}, c_{2}:=\max \{f(\delta):|\delta|=1\}$ exists, and $c_{2} \leq c_{1}$. Moreover, by (4), $\|\ell\| \leq c_{1}<\infty$. Let $\eta \in \mathbb{C}^{n}$ with $|\eta|=1$ and $f(\eta)=c_{2}$. We may assume that $\ell \neq \equiv 0$, i.e., $\|\ell\|>0$, and then $c_{2}>0$ too.

First, let $v \in L^{\infty}(\Omega)$ with $\|v\|_{\infty} \leq 1$ and $\ell(v) \neq 0$, and put $\delta:=\frac{\ell(v)}{|l(v)|}$. Then, by the Cauchy-Schwarz inequality,

$$
|\ell(v)|=\delta^{*} \ell(v)=\int_{\Omega} \delta^{*} K(\omega) v(\omega) d \mu(\omega) \leq \int_{\Omega}\left|K^{*}(\omega) \delta\right| d \mu(\omega)\|v\|_{\infty} \leq c_{2} .
$$

Hence, $\|\ell\| \leq c_{2}$.

Next, let $\tilde{\Omega}:=\left\{\omega \in \Omega: K^{*}(\omega) \eta \neq 0\right\}$ and define

$$
v_{0}(\omega):= \begin{cases}\frac{K^{\bullet}(\omega) \eta}{\left|K^{\bullet}(\omega) \eta\right|} & \text { for } \omega \in \tilde{\Omega} \\ 0 & \text { otherwise }\end{cases}
$$

Then, $v_{0} \in L^{\infty}(\Omega)$ with $\left\|v_{0}\right\|_{\infty}=1($ since $f(\eta)>0)$, and

$$
c_{2}=f(\eta)=\int_{\Omega}\left|K^{*}(\omega) \eta\right| d \mu(\omega)=\eta^{*} \ell\left(v_{0}\right) \leq\|\ell\||\eta|\left\|v_{0}\right\|_{\infty}=\|\ell\| .
$$

Thus, $c_{2} \leq\|\ell\|$, which implies the assertion

The preceding proof also yields the following

Supplement. Suppose that the assumptions of Theorem 1 hold. Then, $\|\ell\|=$ $\left|\ell\left(v_{0}\right)\right|$ for

$$
v_{0}(\omega):= \begin{cases}\frac{K^{*}(\omega) \eta}{\left|K^{*}(\omega) \eta\right|} & \text { if } K^{*}(\omega) \eta \neq 0 \\ 0 & \text { otherwise }\end{cases}
$$

where $\eta \in \mathbb{C}^{n}$ with $|\eta|=1$ and $f(\eta)=\max \{f(\delta):|\delta|=1\}=c_{2}$. Conversely, if $\|\ell\|=\left|\ell\left(v_{0}\right)\right|$ for some $v_{0} \in L^{\infty}(\Omega)$ with $\left\|v_{0}\right\|_{\infty} \leq 1$, then $f(\eta)=c_{2}$ for $\eta=\frac{\ell\left(v_{0}\right)}{\left|\ell\left(v_{0}\right)\right|}$ and $v_{0}(\omega)=\alpha \frac{K^{*}(\omega) \eta}{\left|K^{*}(\omega) \eta\right|}$ a.e. on $\tilde{\Omega}=\left\{\omega \in \Omega: K^{*}(\omega) \eta \neq 0\right\}$ with some $\alpha \in \mathbb{C},|\alpha|=1$.

Remark 1. Of course $\|\ell\| \leq \int_{\Omega}\|K(\omega)\| d \mu(\omega)$, and this inequality is strict in general (as, e.g., for the Stokes kernel) whenever $n \geq 2$. Moreover, if assumption (3) does not hold (but if the matrix elements $k_{\mu \nu}$ are measurable), i.e., $\int_{\Omega}\left|k_{\mu \nu}(\omega)\right| d \mu(\omega)=\infty$ for some indices $\mu$ and $\nu$, then it follows quite easily that $\|\ell\|=\sup \{f(\delta):|\delta|=1\}=\infty$. Hence, the assertion of Theorem 1 remains true in this sense. Note that, of course, all quantities may be real-valued rather than complex-valued, i.e., $\mathbb{C}$ may be replaced by $\mathbb{R}$ throughout the section above. 
Next, suppose that all quantities are real-valued, and let the "extremal function" $v_{0}(\omega)$ and $\eta$ be given as in the Supplement, so that $\ell\left(v_{0}\right)=\lambda \eta$ for $\lambda:=\left|\ell\left(v_{0}\right)\right|=\|\ell\|$. Hence,

$$
\ell\left(v_{0}\right)=\int_{\bar{\Omega}} \frac{K(\omega) K^{T}(\omega)}{\left|K^{T}(\omega) \eta\right|} \eta d \mu(\omega)=\lambda \eta
$$

where $\tilde{\Omega}=\left\{\omega \in \Omega: K^{T}(\omega) \eta \neq 0\right\}$. This formula corresponds to the well-known Lagrange multiplier rule [1: Theorem 7-10] for maximizing $f(\delta)$ under the constraint $|\delta|=1$, because "formal" differentiation implies that

$$
\left.\operatorname{grad} f(\delta)\right|_{\delta=\eta}=\int_{\bar{\Omega}} \frac{K(\omega) K^{T}(\omega)}{\left|K^{T}(\omega) \eta\right|} \eta d \mu(\omega)=\lambda \eta=\left.\lambda \operatorname{grad}\{|\delta|\}\right|_{\delta=\eta}
$$

Theorem 1 can be derived also by abstract arguments from functional analysis. The assertion of Theorem 1 can be reduced to the fact that the norms of a certain linear operator and its adjoint coincide (see [9: Theorem 4.10]), an observation, which is due to $W$. Arendt. In this way Theorem 1 is shown via a "vector-valued" version of the Hahn-Banach theorem (see [9: Theorem 4.3 and the corollary to Theorem 3.3]).

\section{The Stokes kernel}

The basis of this and the next section is the following Stokes-Poisson integral formula [3: Theorem 1].

Lemma 1. For any continuous boundary values $v(\xi) \in C(\partial B)$ there exists a unique Stokes function $v$ in $B$ with $v \in C(B \cup \partial B)$ (where $B$ is defined by (2), and $n \geq 2$ ), and this function is given by

$$
v(x)=\int_{\partial B} S(\xi, x) v(\xi) d \sigma(\xi) \quad \text { for } \quad x \in B
$$

where the Stokes kernel $S(\xi, x)$ is defined by

$$
S(\xi, x):=P(\xi, x) I+\frac{1-|x|^{2}}{2} \int_{0}^{1} H(\xi, t x) d t
$$

with Poisson kernel $P(\xi, x):=\frac{1-|x|^{2}}{\sigma_{n}|\xi-x|^{n}}$ (where $\sigma_{n}=2 \frac{\pi^{n / 2}}{\Gamma(n / 2)}$ is the surface area of the unit sphere $\partial B)$, with Hessian $H$ of the Poisson kernel, i.e., $H(\xi, x)=D_{x}^{2} P(\xi, x)$, and with $(n \times n)$-identity matrix $I$.

The next lemma states basic properties of the Stokes kernel.

Lemma 2. The following assertions hold for all $\xi, \eta \in \partial B, x \in B$ and $n \geq 2$ :

(i) $S(\xi, x)$ is a real and symmetric $(n \times n)$-matrix. 
(ii) $S(\xi, x)=\frac{n\left(1-|x|^{2}\right)}{2 \sigma_{n}} \tilde{S}(\xi, x)$, where

$$
\tilde{S}(\xi, x):=2 \frac{(\xi-x)(\xi-x)^{T}}{|\xi-x|^{n+2}}+n \int_{0}^{1} \frac{(\xi-t x)(\xi-t x)^{T}}{|\xi-t x|^{n+2}} d t-\int_{0}^{1} \frac{d t}{|\xi-t x|^{n}} I .
$$

(iii) $S(\xi, x)$ is invertible, moreover, it possesses one positive eigenvalue and $n-1$ negative eigenvalues.

$$
\text { (iv) } \frac{n(1-|x|)^{2}}{4 \sigma_{n}(1+|x|)^{n}} \leq|S(\xi, x) \eta| \leq n 2^{n-1} P(\xi, x) \text {. }
$$

Proof. Statement (i) follows from definition (6), since $H(\xi, x)$ is a Hessian. Formula (ii) can be derived by straight forward differentiation as follows (compare [3: Lemma 2]):

$$
\begin{aligned}
\sigma_{n} H(\xi, x)= & -\left\{\frac{2}{|\xi-x|^{n}}+n \frac{1-|x|^{2}}{|\xi-x|^{n+2}}\right\} I \\
& -\frac{2 n}{|\xi-x|^{n+2}}\left\{x(\xi-x)^{T}+(\xi-x) x^{T}\right\} \\
& +\frac{n(n+2)}{|\xi-x|^{n+4}}\left(1-|x|^{2}\right)(\xi-x)(\xi-x)^{T}, \\
\frac{d}{d t} \frac{2 t}{|\xi-t x|^{n}}= & \frac{n\left(1-t^{2}|x|^{2}\right)}{|\xi-t x|^{n+2}}-\frac{n-2}{|\xi-t x|^{n}}, \\
\frac{d t}{d t} \frac{2 t(\xi-t x)(\xi-t x)^{T}}{|\xi-t x|^{n+2}}= & (\xi-t x)(\xi-t x)^{T}\left\{\frac{(n+2)\left(1-t^{2}|x|^{2}\right)}{|\xi-t x|^{n+4}}-\frac{n}{|\xi-t x|^{n+2}}\right\} \\
& -2 t \frac{x(\xi-t x)^{T}+(\xi-t x) x^{T}}{|\xi-t x|^{n+2}},
\end{aligned}
$$

and therefore our definition (6) implies that

$$
\begin{aligned}
2 \sigma_{n} S(\xi, x)= & 2 \frac{1-|x|^{2}}{|\xi-x|^{n}} I+\left(1-|x|^{2}\right)\left\{-n \cdot \int_{0}^{1} \frac{d t}{|\xi-t x|^{n}} I-\frac{2}{|\xi-x|^{n}} I\right. \\
& \left.+n^{2} \int_{0}^{1} \frac{(\xi-t x)(\xi-t x)^{T}}{|\xi-t x|^{n+2}} d t+2 n \frac{(\xi-x)(\xi-x)^{T}}{(\xi-x)^{n+2}}\right\}
\end{aligned}
$$

which is assertion (ii).

Now, let $\lambda_{\nu}=\lambda_{\nu}(\xi, x) \quad(1 \leq \nu \leq n)$ denote the eigenvalues of $\tilde{S}(\xi, x)$ for fixed $\xi \in \partial B$ and $x \in B$ : Obviously, every $z \in \mathbb{R}^{n} \backslash\{0\}$ with $z^{T} x=z^{T} \xi=0$ is an eigenvector with corresponding eigenvalue $-\int_{0}^{1} \frac{d t}{|\xi-t x|^{n}}$. Hence, we have with suitable enumeration of the $\lambda_{\nu}$ that

$$
\lambda_{\nu}=-\int_{0}^{1} \frac{d t}{|\xi-t x|^{n}}<0 \quad \text { for } 1 \leq \nu \leq n-2 .
$$


Since trace $H(\xi, x)=\Delta_{x} P(\xi, x)=0$, we obtain from assertion (ii) and (6) that

$$
\operatorname{trace} \frac{n\left(1-|x|^{2}\right)}{2 \sigma_{n}} \tilde{S}(\xi, x)=\operatorname{trace} P(\xi, x) I=n P(\xi, x) .
$$

The idea to consider this trace is due to J. Beurer. Thus, we get that (use also (8))

$$
\sum_{\nu=1}^{n} \lambda_{\nu}=\frac{2}{|\xi-x|^{n}}, \quad \lambda_{n}+\lambda_{n-1}=\frac{2}{|\xi-x|^{n}}+(n-2) \int_{0}^{1} \frac{d t}{|\xi-t x|^{n}}>0 .
$$

Therefore $\lambda_{n}>0$ if $\lambda_{1} \leq \lambda_{2} \leq \ldots \leq \lambda_{n}$, and it follows immediately from assertion (ii) that, for all $1 \leq \nu \leq n$,

$$
-\int_{0}^{1} \frac{d t}{|\xi-t x|^{n}} \leq \lambda_{\nu} \leq\|\tilde{S}(\xi, x)\| \leq \frac{2}{|\xi-x|^{n}}+(n-1) \int_{0}^{1} \frac{d t}{|\xi-t x|^{n}} .
$$

This implies easily the right inequality of statement (iv) (see [3: Proposition 1/(iii)]). For the proof of the other assertions of (iii) and (iv) fix $\xi \in \partial B$ and $x \in B$. If $\xi$ and $x$ are linearly dependent, then

$$
\lambda_{n-1}=-\int_{0}^{1} \frac{d t}{|\xi-t x|^{n}} \quad \text { and } \quad \lambda_{n}=\frac{2}{|\xi-x|^{n}}+(n-1) \int_{0}^{1} \frac{d t}{|\xi-t x|^{n}}
$$

which completes the proof in this case. Hence, suppose that $\xi$ and $x$ are linearly independent. We consider three cases:

Case (i): $\xi^{T} x \leq 0$. Using the formula

$$
\frac{d}{d t} \frac{1}{|\xi-t x|^{n}}=n \frac{x^{T}(\xi-x)}{|\xi-t x|^{n+2}}
$$

we obtain from assertion (ii) via integration by parts

$$
\begin{aligned}
& \xi^{T} \tilde{S}(\xi, x) \xi-\frac{2}{|\xi-x|^{n}}-(n-2) \int_{0}^{1} \frac{d t}{|\xi-t x|^{n}} \\
& \quad=2 \frac{\left(1-\xi^{T} x\right)^{2}-|\xi-x|^{2}}{|\xi-x|^{n+2}}+n \int_{0}^{1} \frac{\left(1-\xi^{T} x\right)^{2}-|\xi-t x|^{2}}{|\xi-t x|^{n+2}} d t+\int_{0}^{1} \frac{d t}{|\xi-t x|^{n}} \\
& \quad=2 \frac{\left(\xi^{T} x\right)^{2}-|x|^{2}}{|\xi-x|^{n+2}}+n \int_{0}^{1} \frac{t^{2}\left(\xi^{T} x\right)^{2}-t \xi^{T} x}{|\xi-t x|^{n+2}} d t+\int_{0}^{1} t \frac{d}{d t} \frac{1}{|\xi-t x|^{n}} d t+\int_{0}^{1} \frac{d t}{|\xi-t x|^{n}} \\
& \quad=\frac{1-2 \xi^{T} x+2\left(\xi^{T} x\right)^{2}-|x|^{2}}{|\xi-x|^{n+2}}+n \int_{0}^{1} \frac{t^{2}\left(\xi^{T} x\right)^{2}-t \xi^{T} x}{|\xi-t x|^{n+2}} d t \\
& \quad \geq \frac{1-|x|^{2}}{|\xi-x|^{n+2}}
\end{aligned}
$$


because $\xi^{T} x \leq 0$. Now, (10) implies

$$
\lambda_{n} \geq \frac{2}{|\xi-x|^{n}}+(n-2) \int_{0}^{1} \frac{d t}{|\xi-t x|^{n}}+\frac{1-|x|^{2}}{|\xi-x|^{n+2}}, \quad \lambda_{n-1} \leq-\frac{1-|x|^{2}}{|\xi-x|^{n+2}}<0 .
$$

Case (ii): $0 \leq \xi^{T} x \leq|x|^{2}$. Using the formula (see (11))

$$
\frac{d}{d t} \frac{\xi-t x}{|\xi-t x|^{n}}=-\frac{x}{|\xi-t x|^{n}}+n \frac{(\xi-t x) x^{T}(\xi-t x)}{|\xi-t x|^{n+2}}
$$

we obtain from (ii) by a simple calculation the identity

$$
\tilde{S}(\xi, x) x=-\xi+\frac{1-|x|^{2}}{|\xi-x|^{n+2}}(\xi-x)
$$

Hence, for $\eta=\frac{x}{|x|}$, we have that

$$
\eta^{T} \tilde{S}(\xi, x) \eta=\frac{1}{|x|^{2}}\left\{-\xi^{T} x+\frac{1-|x|^{2}}{|\xi-x|^{n+2}}\left(\xi^{T} x-|x|^{2}\right)\right\}
$$

and this implies that

$$
\begin{array}{ll}
\lambda_{n-1} \leq-\frac{1}{2} \frac{1-|x|^{2}}{|\xi-x|^{n+2}}<0 & \text { for } 0 \leq \xi^{T} x \leq \frac{1}{2}|x|^{2} \\
\lambda_{n-1} \leq-\frac{1}{2} \quad<0 & \text { for } \frac{1}{2}|x|^{2} \leq \xi^{T} x \leq|x|^{2}
\end{array}
$$

Case (iii): $|x|^{2} \leq \xi^{T} x<|x|$. Let $\eta=\frac{\xi-x}{|\xi-x|}$. We obtain from assertion (ii) via a simple but tedious calculation (use (11) and integration by parts)

$$
\begin{aligned}
\eta^{T} \tilde{S}(\xi, x) \eta-\frac{2}{|\xi-x|^{n}}-(n-2) \int_{0}^{1} \frac{d t}{|\xi-t x|^{n}} \\
=\frac{n}{|\xi-x|^{2}} \int_{0}^{1} \frac{\left\{(\xi-x)^{T}(\xi-t x)\right\}^{2}}{|\xi-t x|^{n+2}} d t-(n-1) \int_{0}^{1} \frac{d t}{|\xi-t x|^{n}} \\
=\int_{0}^{1} \frac{d t}{|\xi-t x|^{n}}+n \int_{0}^{1} \frac{(t-1) x^{T}(\xi-t x)}{|\xi-t x|^{n+2}} d t \\
\quad+n \frac{x^{T}(\xi-x)}{|\xi-x|^{2}} \int_{0}^{1} \frac{(1-t)|\xi-t x|^{2}-(1-t)^{2} x^{T}(\xi-t x)}{|\xi-t x|^{n+2}} d t
\end{aligned}
$$




$$
\begin{aligned}
& =1+\frac{x^{T}(\xi-x)}{|\xi-x|^{2}}\left\{1+(n-2) \int_{0}^{1} \frac{(1-t)}{|\xi-t x|^{n}} d t\right\} \\
& \geq 1+\frac{x^{T}(\xi-x)}{|\xi-x|^{2}} \\
& =\frac{1-\xi^{T} x}{|\xi-x|^{2}} \\
& \geq \frac{1-|x|}{|\xi-x|^{2}}
\end{aligned}
$$

using that $x^{T}(\xi-x) \geq 0$ in the present case. From (9) we obtain similarly as in the case (i) that

$$
\lambda_{n-1} \leq-\frac{1-|x|}{|\xi-x|^{2}}<0 .
$$

Now, the assertion (iii) follows from (8), (12), (14) and (15). Moreover, elementary estimates show that

$$
\min \left\{\left|\lambda_{\nu}\right|: 1 \leq \nu \leq n\right\} \geq \frac{1-|x|}{2(1+|x|)^{n+1}},
$$

which yields the left inequality of (iv) via (ii)

The following lemma follows essentially from Lemma 2/(ii).

Lemma 3. The following statements hold for $n \geq 2$ :

(i) $\int_{\partial B}\left\|S(\xi, x)-S_{0}(\xi, x)\right\| d \sigma(\xi) \rightarrow 0$ as $|x| \rightarrow 1-$, where

$$
S_{0}(\xi, x):=\frac{n\left(1-|x|^{2}\right)}{\sigma_{n}|\xi-x|^{n+2}}(\xi-x)(\xi-x)^{T} .
$$

(ii) $\int_{\partial B}\left\|S_{0}(\xi, x)\right\| d \sigma(\xi)=n$ for all $x \in B$.

(iii) $\int_{\partial B}\|S(\xi, x)\| d \sigma(\xi) \leq \frac{n(n+1)}{2}$ for $|x| \leq 1$, with equality if and only if $x=0$.

Proof. It follows from Lemma $2 /(\mathrm{ii})$ and $\int_{\partial B} P(\xi, x) d \sigma(\xi) \equiv 1$ that

$$
\begin{aligned}
\int_{\partial B}\left\|S(\xi, x)-S_{0}(\xi, x)\right\| d \sigma(\xi) & \leq \frac{n\left(1-|x|^{2}\right)}{2 \sigma_{n}}(n-1) \int_{\partial B} \int_{0}^{1} \frac{d t}{|\xi-t x|^{n}} d \sigma(\xi) \\
& =\frac{n(n-1)}{2}\left(1-|x|^{2}\right) \int_{0}^{1} \frac{d t}{1-t^{2}|x|^{2}} \\
& \rightarrow 0 \text { as }|x| \rightarrow 1-.
\end{aligned}
$$

which yields statement (i). Of course, $\int_{\partial B}\left\|S_{0}(\xi, x)\right\| d \sigma(\xi)=n \int_{\partial B} P(\xi, x) d \sigma(\xi)=n$, and assertion (iii) (compare [3: Proposition 1/(ii)]) follows similarly . 
Remark 2. Let us shortly comment the invertibility of the Stokes kernel. It stands for the fact that any "needle perturbation" of the boundary values has an influence upon the flow at every point in the ball. More precisely: if $\xi_{0} \in \partial B, v_{0} \in \mathbb{R}^{n} \backslash\{0\}$, and $v_{\varepsilon}(\xi)=v_{0}$ for all $\xi \in \partial B$ with $\left|\xi-\xi_{0}\right|<\varepsilon$ and $v_{\varepsilon}(\xi) \equiv 0$ otherwise, then $\lim _{\varepsilon \rightarrow 0} \frac{v_{c}(x)}{\sigma(\varepsilon)}=$ $S\left(\xi_{0}, x\right) v_{0} \neq 0$ for all $x \in B$, where $v_{\varepsilon}(x)=\int_{\partial B} S(\xi, x) v_{\varepsilon}(\xi) d \sigma(\xi)$ and where $\sigma(\varepsilon)$ denotes the surface area of $\left\{\xi \in \partial B:\left|\xi-\xi_{0}\right|<\varepsilon\right\}$. It is, of course, an open problem, whether such a statement is true for Stokes flows not only in balls but in general domains.

\section{Solution of the extremal problem and some asymptotics}

Using the notation of the previous section, in particular the definition of the Stokes kernel by (6), the solution of the extremal problem of the introduction reads as follows.

Theorem 2. Let $n \geq 2$, and define $c_{n}(x)$ and $B$ by (1) and (2), respectively. Then, the supremum defining $c_{n}(x)$ is attained, and

$$
c_{n}(x)=\max _{\delta \in \partial B} \int_{\partial B}|S(\xi, x) \delta| d \sigma(\xi) .
$$

Moreover, $c_{n}(x)=|v(x)|$ for a Stokes function $v$ in $B$ with $v \in C(B \cup \partial B)$ if and only if its boundary values are given by

$$
v(\xi)=\frac{S(\xi, x) \eta}{|S(\xi, x) \eta|} \quad(\xi \in \partial B)
$$

where $\eta \in \partial B$ with $\max _{\delta \in \partial B} \int_{\partial B}|S(\xi, x) \delta| d \sigma(\xi)=\int_{\partial B}|S(\xi, x) \eta| d \sigma(\xi)$.

Proof. The assertions follow immediately from Theorem 1 and its Supplement, because $K(\xi)=S(\xi, x)$ is a continuous, invertible, and symmetric $(n \times n)$-matrix-valued function on $\partial B$ by Lemma 2

Remark 3. Note that the "extremal boundary values" $v(\xi)$, given by $(17)$, satisfy $|v(\xi)|=1$ for all $\xi \in \partial B$. Moreover, formula (17) and the Stokes-Poisson integral formula of Lemma 1 can be used to calculate in addition to the optimal constants $c_{n}(x)$ also the corresponding "extremal flows" $v(x)$ inside the ball $B$.

Rather elementary but extensive estimates and calculations lead to the following corollary, by using Lemma $2 /(\mathrm{ii})$, Lemma 3 , and that $c_{n}:=\sup _{x \in B} c_{n}(x) \geq n$ by $[3$ : Remark 3].

Corollary 1. The constants $c_{n}(x)$, given by (1) or (16), satisfy the following assertions:

(i) $c_{n}(0)=\frac{n \Gamma(n / 2)}{2 \sqrt{\pi} \Gamma((n-1) / 2)} \int_{0}^{\pi}(\sin \varphi)^{n-2}\left\{1+n(n+2) \cos ^{2} \varphi\right\}^{1 / 2} d \varphi$ for $n \geq 2$. $n \geq 3$.

(ii) $\lim _{|x| \rightarrow 1-} c_{n}(x)=d_{n}:=\frac{2}{\sqrt{\pi}} \frac{\Gamma(n / 2+1)}{\Gamma((n+1) / 2)}$ for $n \geq 2$ with $d_{2}=\frac{4}{\pi}$ and $d_{n} \leq \frac{n}{2}$ for 
(iii) $c_{n}=\max _{x \in B} c_{n}(x)$ exists with $n \leq c_{n} \leq \frac{n(n+1)}{2}$ for $n \geq 2$.

(iv) $c_{n}(0) \sim \frac{1}{\sqrt{2 \pi}} n^{3 / 2}$ and $d_{n} \sim \sqrt{\frac{2}{\pi}} n^{1 / 2}$ as $n \rightarrow \infty$.

Proof. In the sequel we shorten some lengthy calculations by omitting elementary but straightforward arguments and by writing down only the main steps of the reasoning.

By Lemma $2 /(\mathrm{ii}), S(\xi, 0)=\frac{n}{2 \sigma_{n}}\left\{(\dot{n}+2) \xi \xi^{T}-I\right\}$. Thus, for all $\delta \in \partial B$,

$$
\begin{aligned}
\int_{\partial B}|S(\xi, 0) \delta| d \sigma(\xi) & =\frac{n}{2 \sigma_{n}} \int_{\partial B}\left\{1+n(n+2)\left(\xi^{T} \delta\right)^{2}\right\}^{1 / 2} d \sigma(\xi) \\
& =\frac{n}{2 \sigma_{n}} \int_{0}^{\pi} \sigma_{n-1}(\sin \varphi)^{n-2}\left\{1+n(n+2) \cos ^{2} \varphi\right\}^{1 / 2} d \varphi
\end{aligned}
$$

and this implies assertion (i) by using (16) of Theorem 2. Moreover, as $n \rightarrow \infty$,

$$
\begin{aligned}
c_{n}(0) & \sim \frac{n \Gamma(n / 2)}{2 \sqrt{\pi} \Gamma((n-1) / 2)} \sqrt{n(n+2)} 2 \int_{0}^{\pi / 2}(\sin \varphi)^{n-2} \cos \varphi d \varphi \\
& =\frac{n \Gamma(n / 2)}{\sqrt{\pi} \Gamma((n-1) / 2)} \frac{\sqrt{n(n+2)}}{n-1} \\
& \sim \frac{n^{3 / 2}}{\sqrt{2 \pi}}
\end{aligned}
$$

by Stirling's formula, which yields the first part of assertion (iv).

By (16) of Theorem 2 (use also Lemma 2), $c_{n}(x)$ is continuous on $B$. Therefore, the existence of $\max _{x \in B} c_{n}(x)$ follows from

$$
n \leq c_{n}=\sup _{x \in B} c_{n}(x) \leq \frac{n(n+1)}{2} \quad \text { (by [3: Theorem } 2 \text { and Remark 3]) }
$$

and from $d_{n}=\lim _{|x| \rightarrow 1-} c_{n}(x)$ with $d_{n}<n$ by assertion (ii). Moreover, assertion (ii) and Stirling's formula imply the second part of (iv).

Hence, it remains to prove (ii). By Lemma $3 /(\mathrm{i})$ and (16),

$$
\lim _{|x| \rightarrow 1^{-}}\left\{c_{n}(x)-\max _{\delta \in \partial B} \int_{\partial B}\left|S_{0}(\xi, x) \delta\right| d \sigma(\xi)\right\}=0 .
$$

Now, fix $\delta \in \partial B$. We may assume that

$$
\begin{array}{ll}
x=(r, 0, \ldots, 0)^{T} & \text { with }|x|=r \in(0,1) \\
\delta=\frac{1}{\sqrt{1+\gamma^{2}}}(1, \gamma, 0, \ldots, 0) & \text { with } \gamma \in(0, \infty) .
\end{array}
$$


Then, by Lemma $3 /(i)$, putting $\xi=\left(\xi_{1}, \ldots, \xi_{n}\right)^{T}$,

$$
\begin{aligned}
\int_{\partial B}\left|S_{0}(\xi, x) \delta\right| d \sigma(\xi) & =\frac{n\left(1-|x|^{2}\right)}{\sigma_{n}} \int_{\partial B} \frac{\left|(\xi-x) \delta^{T}(\xi-x)\right|}{|\xi-x|^{n+2}} d \sigma(\xi) \\
& =\frac{n\left(1-|x|^{2}\right)}{\sigma_{n} \sqrt{1+\gamma^{2}}} \int_{\partial B} \frac{\left|\gamma \xi_{2}+\xi_{1}-r\right|}{\left(1+r^{2}-2 r \xi_{1}\right)^{(n+1) / 2}} d \sigma(\xi)
\end{aligned}
$$

We consider separately the case $n=2$. In that case,

$$
\begin{aligned}
\lim _{|x| \rightarrow 1-} & \int_{\partial B}\left|S_{0}(\xi, x) \delta\right| d \sigma(\xi) \\
& =\lim _{r \rightarrow 1-} \frac{2(1-r)(1+r)}{2 \pi \sqrt{1+\gamma^{2}}} \int_{-\pi}^{\pi} \frac{|\gamma \sin \varphi+\cos \varphi-r|}{\left(1+r^{2}-2 r \cos \varphi\right)^{3 / 2}} d \varphi \\
& =\lim _{r \rightarrow 1-} \frac{2(1-r)}{\pi \sqrt{1+\gamma^{2}}} \int_{-\pi}^{\pi} \frac{|\gamma \varphi+1-r|}{\left((1-r)^{2}+\varphi^{2}\right)^{3 / 2}} d \varphi \\
& \left.=\frac{2}{\pi \sqrt{1+\gamma^{2}}} \int_{-\infty}^{\infty} \frac{|\gamma t+1|}{\left(1+t^{2}\right)^{3 / 2}} d t \quad \text { (by substituting } \varphi(1-r) \xi\right) \\
= & \frac{4}{\pi}
\end{aligned}
$$

uniformly for $0<\gamma<\infty$. Hence, $\lim _{|x| \rightarrow 1-} c_{2}(x)=\frac{4}{\pi}$, which is assertion (ii) in this case.

Now, let $n \geq 3$. Then, by the calculation above,

$$
\begin{aligned}
\lim _{|x| \rightarrow 1-} & \int_{\partial B}\left|S_{0}(\xi, x) \delta\right| d \sigma(\xi) \\
= & \lim _{r \rightarrow 1-} \frac{n\left(1-r^{2}\right)}{\sigma_{n} \sqrt{1+\gamma^{2}}} \int_{0}^{\pi} \int_{-\pi / 2}^{\pi / 2} \sigma_{n-2}(\sin \vartheta \cos \varphi)^{n-3} \\
& \times \frac{|\gamma \sin \vartheta \sin \varphi+\cos \vartheta-r|}{\left(1+r^{2}-2 r \cos \vartheta\right)^{(n+1) / 2}} \sin \vartheta d \varphi d \vartheta \\
= & \lim _{r \rightarrow 1-} \frac{n(n-2)(1-r)}{\pi \sqrt{1+\gamma^{2}}} \int_{0}^{\pi / 2} \int_{-\pi / 2}^{\pi / 2} \frac{(\cos \varphi)^{n-3} \vartheta^{n-2}|\gamma \vartheta \sin \varphi+1-r|}{\left((1-r)^{2}+\vartheta^{2}\right)^{(n+1) / 2}} d \varphi d \vartheta \\
= & (\text { by substituting } \vartheta=(1-r) t) \\
& \frac{n(n-2)}{\pi \sqrt{1+\gamma^{2}}} \int_{-\pi / 2}^{\pi / 2}(\cos \varphi)^{n-3}\left\{\int_{0}^{\infty} \frac{|1+\gamma t \sin \varphi| t^{n-2}}{\left(1+t^{2}\right)^{(n+1) / 2}} d t\right\} d \varphi
\end{aligned}
$$


$=$ (by integration by parts and by explicit integration, respectively)

$$
\begin{aligned}
& \frac{n(n-2)}{\pi \sqrt{1+\gamma^{2}}} \int_{0}^{\pi / 2}(\cos \varphi)^{n-3} \\
& \times\left\{2 \gamma \sin \varphi \int_{0}^{\gamma \sin \varphi} \frac{d \tau}{\left(1+\tau^{2}\right)^{(n+1) / 2}}+2 \int_{\gamma \sin \varphi}^{\infty} \frac{\tau d \tau}{\left(1+\tau^{2}\right)^{(n+1) / 2}}\right\} d \varphi \\
= & \frac{2 n(n-2)}{\pi \sqrt{1+\gamma^{2}}} \int_{0}^{\pi / 2} \frac{(\cos \varphi)^{n-3}}{\left(1+\gamma^{2} \sin ^{2} \varphi\right)^{(n+1) / 2}}\left\{\frac{\gamma^{2} \cos ^{2} \varphi}{n-2}+\frac{1+\gamma^{2} \sin ^{2} \varphi}{n-1}\right\} d \varphi \\
= & \left(\text { by substituting } \sqrt{1+\gamma^{2}} \tan \varphi=t\right) \\
& \frac{2 n(n-2)}{\pi\left(1+\gamma^{2}\right.}\left\{\frac{\gamma^{2}}{n-2} \int_{0}^{\infty} \frac{d t}{\left(1+t^{2}\right)^{(n+1) / 2}}+\frac{1}{n-1} \int_{0}^{\infty} \frac{d t}{\left(1+t^{2}\right)^{(n-1) / 2}}\right\} \\
= & \frac{2 n}{\pi} \int_{0}^{\infty} \frac{d t}{\left(1+t^{2}\right)^{(n+1) / 2}}
\end{aligned}
$$

uniformly for $0<\gamma<\infty$. Hence, for $n \geq 3$,

$$
\lim _{|x| \rightarrow 1^{-}} c_{n}(x)=d_{n}=\frac{2 n}{\pi} \int_{0}^{\infty} \frac{d t}{\left(1+t^{2}\right)^{(n+1) / 2}} .
$$

The recursion $\frac{d_{n}}{n}=\frac{d_{n-2}}{n-2} \frac{n-2}{n-1}$ yields easily the assertions of statement (ii). Observe that the limits do not depend on $\gamma$, i.e., on $\delta \in \partial B$, in both cases, i.e., for $n=2$ and $n \geq 3$

Remark 4. For dimensions $n=2$ and $n=3$ we have by (i) and (ii) of Corollary 1 the following explicit constants:

$$
\begin{aligned}
& c_{2}(0)=\frac{1}{\pi} \int_{0}^{\pi} \sqrt{1+8 \cos ^{2} \varphi} d \varphi=2.127 \ldots \\
& c_{3}(0)=3+\frac{3}{4 \sqrt{15}} \log (4+\sqrt{15})=3.399 \ldots, \quad d_{2}=\frac{4}{\pi}, \quad d_{3}=\frac{3}{2} .
\end{aligned}
$$

As already mentioned in the introduction, numerical calculations by $A$. Peyerimhoff in dimensions $n=2,3,4,5,6$ suggest the conjecture that

$$
c_{n}=\max _{x \in B} c_{n}(x)=c_{n}(0) \quad \text { for all } n \geq 2 \text {. }
$$

This does hold for $n=2$, i.e., $c_{2}=c_{2}(0)=2.127 \ldots$, as is shown in [5]. Moreover, the numerical results indicate that $c_{n}(x)=c_{n}(|x|)$ is a decreasing and concave function on $[0,1)$ for all $n \geq 2$. 


\section{Further applications of the general theorem}

In this section we use Theorem 1 to establish two inequalities. The first application concerns complex functions on the unit circle.

Proposition 1. Let be given complex-valued functions $a$ and $b$ on the unit circle $\mathcal{C}=\{\xi \in \mathbb{C}:|\xi|=1\}$ with $a, b \in L^{1}(\mathcal{C})$. Then,

$$
\max \left\{\left|\int_{\mathcal{C}}\{a(\xi) v(\xi)+b(\xi) \bar{v}(\xi)\} \frac{d \xi}{\xi}\right|: v \in L^{\infty}(\mathcal{C}) \text { with }\|v\|_{\infty} \leq 1\right\}
$$

exists, and it equals

$$
\max _{\delta \in \mathcal{C}} \int_{\mathcal{C}}|a(\xi)+\delta \bar{b}(\xi)| \frac{d \xi}{i \xi}
$$

(Note that $\int_{\mathcal{C}} f(\xi) \frac{d \xi}{i \xi}=\int_{0}^{2 \pi} f\left(e^{i \varphi}\right) d \varphi$ for any function $f$ on $\mathcal{C}$.)

Proof. We split the functions $a, b$ and $v$ into their real and imaginary parts, i.e.,

$$
a=\alpha_{1}+i \alpha_{2}, \quad b=\beta_{1}+i \beta_{2}, \quad v=u+i w,
$$

so that

$$
a v+b \bar{v}=\gamma_{1}+i \gamma_{2} \quad \text { with }\left(\begin{array}{c}
\gamma_{1} \\
\gamma_{2}
\end{array}\right)=K\left(\begin{array}{l}
u \\
w
\end{array}\right) \text { for } K=\left(\begin{array}{cc}
\alpha_{1}+\beta_{1} & \beta_{2}-\alpha_{2} \\
\alpha_{2}+\beta_{2} & \alpha_{1}-\beta_{1}
\end{array}\right)
$$

Then $K: \Omega=\mathcal{C} \rightarrow \mathbb{R}^{2 \times 2}$ satisfies assumption (3) of Theorem 1. Hence, our assertion follows from Theorem 1 , because $\left|K^{*} \delta\right|=\left|K^{T} \delta\right|=|\bar{a} \delta+b \bar{\delta}|=\left|a+\bar{b} \delta^{2}\right|$ for $\delta \in \mathcal{C}$

Remark 5. If the maximum in (19) is attained for $\delta=\eta \in \mathcal{C}$, then according to the Supplement of Theorem 1, the maximum in (18) is attained for the extremal function $v_{0}$ given by

$$
v_{0}(\xi)= \begin{cases}\frac{\bar{a}(\xi)+b(\xi) \bar{\eta}}{|\bar{a}(\xi)+b(\xi) \bar{\eta}|} & \text { if } \bar{a}(\xi)+b(\xi) \bar{\eta} \neq 0 \\ 0 & \text { otherwise. }\end{cases}
$$

An immediate consequence of Proposition 1 is the following

Application. Let be given trigonometric polynomials

$$
a(\xi)=\sum_{k=-n}^{n} a_{k} \xi^{k} \quad \text { and } \quad b(\xi)=\sum_{k=-n}^{n} b_{k} \xi^{k} \quad \text { for } \xi=e^{i \varphi} \in \mathcal{C}
$$

Then, for every function $v \in L^{\infty}(\mathcal{C})$ with Fourier coefficients

$$
c_{k}=\frac{1}{2 \pi} \int_{0}^{2 \pi} v\left(e^{i \varphi}\right) e^{-i k \varphi} d \varphi \quad \text { for } k \in \mathbb{Z}
$$


the inequality

$$
\left|\sum_{k=-n}^{n}\left\{a_{-k} c_{k}+b_{k} \bar{c}_{k}\right\}\right| \leq c\|v\|_{\infty}
$$

holds for $c=\max _{\delta \in \mathcal{C}} \frac{1}{2 \pi} \int_{0}^{2 \pi}\left|a\left(e^{i \varphi}\right)+\delta \vec{b}\left(e^{i \varphi}\right)\right| d \varphi$, and this constant is optimal.

Observe that the functions $a(\xi) \equiv 1$ and $b(\xi)=2 \xi^{2}$ lead to the constant $c=c_{2}(0)=$ $2.127 \ldots$ from Remark 4, which follows from the Stokes-Poisson integral formula in the plane with complex notation (see [4: Theorem 1] for $z=0$ ).

The other application of Theorem 1 concerns an inequality in matrix analysis. It follows immediately from Theorem 1 for the special case that the abstract integral reduces to a finite sum.

Proposition 2. Let be given matrices $A_{1}, \ldots, A_{m} \in \mathbb{C}^{n \times n}$. Then,

$$
\max \left\{\left|\sum_{k=1}^{m} A_{k} c_{k}\right|: c_{k} \in \mathbb{C}^{n},\left|c_{k}\right| \leq 1\right\}=\max \left\{\sum_{k=1}^{m}\left|A_{k}^{*} \delta\right|: \delta \in \mathbb{C}^{n},|\delta|=1\right\}
$$

\section{References}

[1] Apostol, T. M.: Mathematical Analysis. Reading: Addison-Wesley 1965.

[2] Galdi, G. P.: An Introduction to the Mathematical Theory of the Navier-Stokes Equations. Volume I: Linearized Steady Systems. New York: Springer-Verlag 1994.

[3] Kratz, W.: The maximum modulus theorem for the Stokes system in a ball. Math. Z. 226 (1997), $389-403$.

[4] Kratz, W.: On the maximum modulus theorem for Stokes functions. Appl. Anal. 58 (1995), 293 - 302.

[5] Krat2, W.: An extremal problem for Stokes functions in the plane. Analysis 17 (1997), $219-225$.

[6] Pipher, J. and G. Verchota: A maximum principle for biharmonic functions in Lipschitz and $C^{1}$ domains. Comment. math. Helv. 68 (1993), $385-414$.

[7] Maremonti, P. and R. Russo: On the maximum modulus theorem for the Stokes system. Ann. Scuola Norm. Sup. Pisa 21 (1994), 629 - 643.

[8] Naumann, J.: On a maximum principle for weak solutions of the stationary Stokes system. Ann. Scuola Norm. Pisa 15 (1988), $149-168$.

[9] Rudin, W.: Functional Analysis. New York: McGraw-Hill 1973.

[9] Varnhorn, W.: The Stokes equations. Berlin: Akademie Verlag 1994. 